\title{
Transcriptional profiling in the livers of rats after hypobaric hypoxia exposure
}

\author{
Zhenguo Xu ${ }^{\text {Equal first author, } 1,2}$, Zhilong Jia ${ }^{\text {Equal first author, } 1,2}$, Jinlong Shi ${ }^{1,2}$, Zeyu Zhang ${ }^{1,2}$, Xiaojian Gao ${ }^{1,2}$, Qian Jia ${ }^{2}$, Bohan \\ Liu $^{2}$, Jixuan Liu ${ }^{1}$, Chunlei Liu ${ }^{1}$, Xiaojing Zhao ${ }^{\text {Corresp., } 1,2}$, Kunlun He ${ }^{\text {Corresp. } 1}$ \\ 1 Laboratory of Translational Medicine, Chinese PLA General Hospital, Beijing, China \\ 2 Beijing Key Laboratory of Chronic Heart Failure Precision Medicine, Chinese PLA General Hospital, Beijing, China \\ Corresponding Authors: Xiaojing Zhao, Kunlun He \\ Email address: xjingzhao@126.com, kunlunhe@plagh.org
}

Ascent to high altitude feels uncomfortable in part because of a decreased partial pressure of oxygen due to the decrease in barometric pressure. The molecular mechanisms causing injury in liver tissue after exposure to a hypoxic environment are widely unknown. The liver must physiologically and metabolically change to improve tolerance to altitudeinduced hypoxia. Since the liver is the largest metabolic organ and regulates many physiological and metabolic processes, it plays an important part in high altitude adaptation. The cellular response to hypoxia results in changes in the gene expression profile. The present study explores these changes in a rat model. To comprehensively investigate the gene expression and physiological changes under hypobaric hypoxia, we used genome-wide transcription profiling. Little is known about the genome-wide transcriptional response to acute and chronic hypobaric hypoxia in the livers of rats. In this study, we carried out RNA-Sequencing (RNA-Seq) of liver tissue from rats in three groups, normal control rats (L), rats exposed to acute hypobaric hypoxia for 2 weeks (W2L) and rats chronically exposed to hypobaric hypoxia for 4 weeks (W4L), to explore the transcriptional profile of acute and chronic mountain sickness in a mammal under a controlled time-course. We identified 497 differentially expressed genes between the three groups. A principal component analysis revealed large differences between the acute and chronic hypobaric hypoxia groups compared with the control group. Several immunerelated and metabolic pathways, such as cytokine-cytokine receptor interaction and galactose metabolism, were highly enriched in the KEGG pathway analysis. Similar results were found in the Gene Ontology analysis. Cogena analysis showed that the immunerelated pathways were mainly upregulated and enriched in the acute hypobaric hypoxia group. 


\section{Transcriptional profiling in the livers of Rats after Hypobaric Hypoxia Exposure}

Zhenguo $\mathrm{Xu}^{1,2}$, Zhilong Jia ${ }^{1,2}$, Jinlong Shi ${ }^{1,2}$, Zeyu Zhang ${ }^{1,2}$, Xiaojian Gao ${ }^{1,2}$, Qian Jia ${ }^{1,2}$, Bohan Liu $^{1,2}$, Jixuan Liu ${ }^{1,2}$, Chunlei Liu ${ }^{1,2}$, Xiaojing Zhao ${ }^{1,2}$, Kunlun $\mathrm{He}^{1,2}$

1 Laboratory of Translational Medicine, Chinese PLA General Hospital, Beijing, China

2 Beijing key laboratory of chronic heart failure precision medicine, Chinese PLA General Hospital, Beijing, China

Corresponding Author:

Kunlun $\mathrm{He}^{1,2}$, Xiaojing Zhao ${ }^{1,2}$

No. 28 Fuxing Road, 100853, Beijing

Email address: kunlunhe301@plagh.org

\section{ABSTRACT}

Ascent to high altitude feels uncomfortable in part because of a decreased partial pressure of oxygen due to the decrease in barometric pressure. The molecular mechanisms causing injury in liver tissue after exposure to a hypoxic environment are widely unknown. Since the liver is the largest metabolic organ and regulates many physiological and metabolic processes, it plays an important part in high altitude adaptation. The liver must physiologically and metabolically change to improve tolerance after hypobaric hypoxia exposure. To comprehensively investigate the gene expression and physiological changes under hypobaric hypoxia, we used genome-wide transcription profiling.

Little is known about the genome-wide transcriptional response to acute and chronic hypobaric hypoxia in the livers of rats. In this study, we carried out RNA-Sequencing (RNA-Seq) of liver tissue from rats in three groups, normal control rats (L), rats exposed to acute hypobaric hypoxia for 2 weeks (W2L) and rats chronically exposed to hypobaric hypoxia for 4 weeks (W4L), to explore the transcriptional profile of acute and chronic mountain sickness in a mammal under a controlled time-course. We identified 497 differentially expressed genes between the three groups. A principal component analysis revealed large differences between the acute and chronic hypobaric hypoxia groups compared with the control group. Several immune-related and metabolic pathways, such as cytokine-cytokine receptor interaction and galactose metabolism, were highly enriched in the KEGG pathway analysis. Similar results were found in the Gene Ontology analysis. Cogena analysis showed that the immune-related pathways were mainly upregulated and enriched in the acute hypobaric hypoxia group. 
33 INTRODUCTION

34 The defining feature of a high altitude environment is sustained hypobaric hypoxic conditions.

35

36

37

As terrestrial altitude increases, although the atmospheric proportion of oxygen remains constant at $21 \%$, barometric pressure falls. In the meantime, the partial pressure of oxygen decreases at high altitude, giving rise to hypobaric hypoxia. For humans ascending to a high altitude, the lower partial pressure of inspired oxygen leads to a reduction in the oxygen content of arterial blood, called systemic hypoxemia, and subsequently leads to tissue hypoxia with diminished cellular/mitochondrial oxygen availability (Murray 2016). Accordingly, exposure to high altitudes may result in acute mountain sickness (AMS), a syndrome causing headaches and other symptoms occurring at altitudes $>2500$ meter (Honigman et al. 1993; Roach et al. 2018). Although it makes intuitive sense that arterial oxygen saturation should correlate with AMS symptoms, several studies have not found a significant association, at least at moderate altitudes similar to those at the Pace Laboratory at the Barcroft Station (3800 meter) (Guo et al. 2014; O'Connor et al. 2004). The majority of the world's population inhabits regions below 1000 meter elevation; nevertheless, our species has a remarkable capacity for hypoxia tolerance. Most notably, over tens of thousands of years, some high-altitude populations have adapted to life in this environment, with genetic signatures revealing natural selection around hypoxia-sensing pathways (Bigham \& Lee 2014). Approximately 140 million people live above 2500 meter, but approximately 40 million others venture into high altitude regions each year for work or leisure (Weil et al. 2007). These lowlanders experience a physiological response upon ascent, which has been well documented elsewhere and includes ventilatory, cardiovascular and erythropoietic factors. Even with adequate acclimatization, metabolic and physiological functions can be affected as the body reestablishes homeostasis under the hypoxic conditions. Energy metabolism is altered in heart and skeletal muscle. In the hypoxic mouse heart, decreased expression of proliferator-activated receptor alpha (PPAR $\alpha$ ) and its targets lowers fatty acid $\beta$-oxidation (FAO) capacity and represents a vital mechanism to conserve energetics and prevent hypoxic injury. In lowlander skeletal muscle, ATP and PCr levels fall at altitude and this loss continues over time. It is suggesting that the suppression of ATP supply is not met with a comparable downregulation of ATP demand at altitude (Hochachka et al. 1996). In a study of Sherpa cardiac energy metabolism, carried out at sea level, a low cardiac phosphocreatine-to-ATP ratios (PCr/ATP) ratio was seen in comparison with lowlanders and this persisted even as the Sherpas acclimatized to sea level. Many other studies have also reported a downregulation of the expression and/or activity of FAO enzymes, many of which are PPAR $\alpha$ targets, both in human muscle at altitude and in the heart and skeletal muscle of hypoxic rodents (Horscroft et al. 2017). This might indicate a failure to fully compensate for the lower oxygen availability or may itself form part of the acclimatization process, but it involves changes in gene expression and thus appears to be a regulated response. The positive aspects of high altitude acclimatization most notably decrease susceptibility to acute mountain sickness. However, the less well-understood aspect of high altitude deterioration is characterized by AMS symptoms including lethargy, fatigue and muscle wasting after prolonged exposure to extreme high altitude ( $>5500$ meter)

Peer) reviewing PDF | (2018:10:31961:1:3:ACCEPTED 14 Jan 2019) 
73 (Ward 1954). However, hypoxia is not only stress encountered at high altitude (Weil et al. 2007).

74

Furthermore, hypoxia has been indicated to trigger vascular inflammation, which leads to increased vascular permeability, leukocyte adherence, and leukocyte emigration (Jung et al. 2012; Lam et al. 2012a; Wood et al. 2000). Exposure to hypoxia promotes the expression of transcription factors, including nuclear factor $\mathrm{NF} \kappa \mathrm{B}(\mathrm{NF}-\kappa \mathrm{B})$, which plays a central role in stimulating the release of proinflammatory cytokines (Taylor 2008). Interleukin-1beta (IL-1 $\beta$ ), IL-6, and tumor necrosis factor- $\alpha(\mathrm{TNF}-\alpha)$ have been suggested to increase under hypoxic conditions (Dosek et al. 2007; Seys et al. 2013). These proinflammatory cytokines are released by activated $\mathrm{T}$ cells and macrophages. However, in the literature (Akopian et al. 2002; Bailey et al. 2004), researchers have found that the elevation of these proinflammatory cytokines was not significant, which encouraged us to further study expression changes in tissues due to hypobaric hypoxia with the use of RNA-Seq analysis (Wang et al. 2018). Different tissues of the organism are required to diverse energy requirements under various ranges of oxygen concentrations (Pompella \& Corti 2015). As the largest metabolic organ in the body, the liver perform an important and complex biological functions under different oxygen concentrations. It has an important role in the metabolism of all kinds of component that are essential for survival, including carbohydrates, proteins, lipids etc. The hypoxia effect on different hepatocellular carcinoma cell lines have been analyzed before (Bonewald 1999; Ivanova et al. 2014; Kaelin \& Ratcliffe 2008). However, no in vivo studies have been conducted on the effect of hypobaric hypoxia exposure for 2 to 4 weeks on gene expression in the liver.

\section{MATERIALS \& METHODS}

Animals and treatment

Adult Sprague-Dawley male rats were randomly allocated to 3 groups (4 animals per group) containing normal rats (L), rats exposed to hypobaric hypoxia for 2 weeks (W2L) and rats exposed to chronic hypobaric hypoxia for 4 weeks (W4L), as suggest in the article of $\mathrm{Ni}$ et al (Ni et al. 2014). Rats in the W2L and W4L groups were exposed to a simulated altitude atmosphere with 5500 meters $(380 \mathrm{mmHg})$, implemented by a FLYDWC50-1C low pressure hypoxic experimental cabin (Guizhou Fenglei Air Ordnance LTD, China). However, a rat in the acute exposure group died prior to the completion of the experiment. During breeding and experimental procedures, animals in both groups were housed in the same density per cage at a controlled ambient temperature of $25 \pm 2^{\circ} \mathrm{C}$ and $50 \pm 10 \%$ relative humidity with a $12 \mathrm{~h}$ light/12h dark cycle. Rats were given standard rodent chow and water ad libitum. Following overnight fasting, rats were sacrificed under anesthesia with $10 \%$ chloral hydrate $(0.4 \mathrm{ml} / 100 \mathrm{~g}$ body weight, IP). The piece of right robe of liver was snap-frozen in liquid nitrogen and then stored at $-80^{\circ} \mathrm{C}$ until analysis anesthesia with $10 \%$ chloral hydrate $(0.4 \mathrm{ml} / 100 \mathrm{~g}$ body weight, IP). Control rats $(\mathrm{L})$ were anesthetized and sacrificed on day 1 and processed in the same manner as described above. Chinese PLA General Hospital Animal ethics committee provided full approval for this research (2017-X13-05).

RNA quantification and qualification

Peer) reviewing PDF | (2018:10:31961:1:3:ACCEPTED 14 Jan 2019) 
112 RNA degradation and contamination were assessed on 1\% agarose gels. RNA purity was

113 checked using the NanoPhotometer ${ }^{\circledR}$ spectrophotometer (IMPLEN, CA, USA). RNA

114 concentration was measured using a Qubit ${ }^{\circledR}$ RNA Assay Kit in a Qubit ${ }^{\circledR}$ 2.0 Fluorometer (Life

115 Technologies, CA, USA). RNA integrity was assessed using the RNA Nano 6000 Assay Kit

116 from the Bioanalyzer 2100 system (Agilent Technologies, CA, USA).

117 Library preparation for liver Transcriptome sequencing

118 The input material for the RNA preparations used amount of $1 \mu \mathrm{g}$ of RNA per sample. Following

119 the manufacturer's recommendations, the sequencing libraries were generated with a NEBNext ${ }^{\circledR}$

120 UltraTM RNA Library Prep Kit for Illumina ${ }^{\circledR}$ (NEB, USA) and the index codes were added to

121 attribute sequences to per sample. Simply, The poly-T oligo-attached magnetic beads were used

122 to mRNA purified from total RNA. Divalent cations were used in NEBNext First-Strand

123 Synthesis Reaction Buffer (5X) to break at elevated temperatures. The first strand cDNA was

124 synthesized using a random hexamer primer and M-MuLV reverse transcriptase ( $\mathrm{RNase}^{-}$).

125 Subsequent use of DNA polymerase I and RNase $\mathrm{H}$ for second strand cDNA synthesis. The

126 remaining overhangs are converted to blunt ends by exonuclease/polymerase activity. After

127 adenylation at the 3' end of the DNA fragment, NEBNext Adapters with a hairpin loop structure

128 were ligated to prepare for hybridization. To preferentially select cDNA fragments of

129 approximately 250-300 bp in length, library fragments were purified using the AMPure XP

130 system (Beckman Coulter, Beverly, USA).

131 Then, the size-ligated linker-ligated cDNA was used at $37^{\circ} \mathrm{C}$ for 15 minutes and then at $95^{\circ} \mathrm{C}$ for

1325 minutes at $95^{\circ} \mathrm{C}$ with $3 \mu \mathrm{l}$ of USER Enzyme (NEB, USA). Subsequently, PCR was carried out

133 using Phusion High-Fidelity DNA polymerase, universal PCR primers and index (X) primers.

134 Finally, the PCR product (AMPure XP system) was purified and library quality was assessed on

135 an Agilent Bioanalyzer 2100 system.

136 Clustering and sequencing

137 Indicated by the manufacturer's instructions, indexed samples was clustered on the cBot Cluster

138 Generation System with the TruSeq PE Cluster Kit v3-cBot-HS (Illumina). Library preparations

139 were sequenced on an Illumina Hiseq platform and generated $125 \mathrm{bp} / 150 \mathrm{bp}$ paired-end reads after

140 cluster generation.

141 Data Analysis

142 Quality control

143 In this step, clean data is obtained by deleting reads containing adapters, readings containing

144 ploy-N, and low quality reads from raw data. At the same time, the Q20, Q30 and GC contents of

145 the cleaning data were calculated. All downstream analyses are based on high quality cleaning

146 data. Raw fastq data were first processed by an internal Perl script. In this step, clean data were

147 obtained by deleting reads containing adapters, reads containing a ploy- $\mathrm{N}$ and low quality reads

148 from the raw reads. In the meantime time, the Q20, Q30 and GC content of the clean data were

149 respectively calculated. The high-quality clean data were used in the downstream analyses.

150 Mapping reads to the rat reference genome

151 Reference genome and gene annotation files were obtained directly from the genome website.

152 The paired end clean readings were aligned to the reference genome with Hisat $2 \mathrm{v} 2.0 .4$. Hisat2

Peer) reviewing PDF | (2018:10:31961:1:3:ACCEPTED 14 Jan 2019) 
153 can generate a splice junctions database based on the gene annotation file and can obtain better

154

155

156

157

158

159

160

161

162

163

164

165

166

167

168

169

170

171

172

173

174

175

176

177

178

179

180

181

182

183

184

185

186

187

188

189

190

191

192

mapping results than other non-splicing mapping tools.

Differential expression analysis

The raw read counts of each sample were achieved using HTSeq v 0.9.1(LG et al. 2012). The normalized gene expression data were obtained using the calcNormFactors function from the edgeR package and voom function from the limma package. The L, W2L and W4L groups were compared using the limma package to obtain the differentially expressed genes (DEGs), filtered with the criteria that the corrected p-value was less than 0.05 and the minimum absolute log fold change of any three comparisons (W2L versus L, W4L versus W2L and W4L versus W2L) was more than 2.

Functional analysis and gene set co-expression analysis of the DEGs

The clusterProfiler packages were used to implement the KEGG and Gene Ontology analysis of the DEGs (Yu et al. 2012). The default parameters were used in our analyses. Co-expression pathway analysis was done using the cogena package (Jia et al. 2016).

\section{RESULTS}

Differential expression analysis between the three groups

Clustering and principal component analysis of the samples based on the differentially expressed genes showed that responses to acute and chronic hypobaric hypoxia were different. A total of

497 DEGs were obtained via differential expression analysis (see supplemental Table S1). A heat map of these genes between the three groups is presented with hierarchical clustering of the samples and genes in Fig.1. Compared with the L control group, the expression patterns of differentially expressed genes in the W2L and W4L groups were largely different from each other, though they represented acute and chronic hypobaric hypoxia. As a result, the W4L and L groups were clustered more closely than the W2L and W4L groups, indicating a large difference between the W2L and W4L groups.

The principal component analysis of these samples using all the DEGs further verified this observation, as shown in Fig.2. Briefly, the 3 groups were distinctly separated while samples in the same group were clustered, indicating that the rat models of acute and chronic hypobaric hypoxia were successful. The first principal component explained approximately $50 \%$ of the gene expression signatures and the W2L were highly separated from the L and W4L groups along the $\mathrm{x}$-axis. Moreover, the W4L and L groups were distinct from each other in the second principal component, explaining approximately $20 \%$ of the variation.

Immune-related pathways are highly enriched in a hypobaric hypoxia environment

To explore the biological function of the DEGs responding to hypobaric hypoxia, we used KEGG pathway and Gene Ontology enrichment analyses. Several innate immune pathways, including cytokine-cytokine receptor interaction, the IL-17 signaling pathway, the TNF signaling pathway and the chemokine signaling pathway, were highly enriched in the pathway analysis (Fig.3). Additionally, some metabolic pathways, such as mineral absorption and galactose metabolism, were enriched as well. It seems that immune-related pathways represented in the DEGs are involved in the response and/or adaptation to hypobaric hypoxia.

Peer) reviewing PDF | (2018:10:31961:1:3:ACCEPTED 14 Jan 2019) 
193 We then used the Gene Ontology analysis to annotate the DEGs affected by hypobaric hypoxia

194 (Fig. 4). Concerning molecular function, the GO analysis indicated that some migration and 195 chemotaxis ontologies, such as leukocytes and granulocytes, were significantly enriched. For

196 biological processes, receptor and ligand activity ontologies, especially cytokines and 197 chemokines, were enriched. For cellular components, the GO analysis indicated that the DEGs 198 were active in the extracellular matrix. The GO analysis verified the results of the KEGG 199 pathway analysis.

200 Co-expression pathway analysis

201 Three different co-expression patterns were observed in the co-expression analysis (Fig. 5). Co202 expression analysis has the ability to cluster DEGs that possess the same or similar functions, 203 which supplies a way to refine the DEGs and pathways. Most of the 244 genes in the first cluster 204 were upregulated in week 2 but were downregulated in week 4 Genes in the second cluster, 205 containing 148 genes, were upregulated until week 4.Most genes in the third cluster were 206 downregulated in week 2 and then upregulated in week 4.

207 Pathway analysis for each co-expression cluster was done using the cogena bioconductor 208 package, a tool for gene set co-expression analysis implemented by the authors. For the co209 expression analysis of the DEGs, the k-means clustering method and three clusters for analysis 210 were selected based on the cogena manual. Interestingly, immune-related pathways, such as the 211 cytokine-cytokine receptor interaction pathway and JAK-STAT signaling pathway, were 212 enriched only in the first cluster but not in the other two clusters (Fig.6). Combining this with the 213 regulation direction of the genes in this cluster, it seems that immune-related pathways function 214 in the first 2 weeks but not in week 4.

\section{DISCUSSION}

216 This paper lays the first foundations for understanding transcriptional and signaling pathway 217 changes in rat liver tissue under acute and chronic hypobaric hypoxia. These findings suggest 218 that immune-related pathways play a key role in hypobaric hypoxia exposure and in the 219 mechanistic differences between acute and chronic mountain sickness. The former study found an abundance of differentially expressed genes related to the immune system vary with an altitude of exposure to hypoxia $(1400 \mathrm{~m}, 3000 \mathrm{~m}, 4500 \mathrm{~m})$. Unlikely, the immune system is not typically viewed as important under hypoxia in this study (Baze et al. 2010). However, there is growing evidence that hypoxia may affect the immune system. Several studies of humans at high-altitude indicate perturbations of the immune system, especially about in circulating blood leukocytes. More studies suggest that hypoxia has a pro-inflammatory effect on macrophages, neutrophils and other white blood cells, and has anti-inflammatory effects on certain lymphocytes (Bosco et al. 2006; Walmsley et al. 2005). Furthermore, there is growing evidence that HIF is a key regulator of many immunological processes.

The present findings were obtained by comparing the gene expression profiles of liver tissue under high altitude conditions for 2 to 4 weeks with liver tissue under normal altitude conditions, providing clues to the molecular pathogenesis of this condition. Genome-wide transcriptional analysis suggests that hypoxia-induced proinflammatory cytokines and chemokines lead to liver 
233 injury in week 2 . The study provides important information on the molecular mechanism causing 234 liver injury at high altitudes and lays a foundation for subsequent gene validation and functional 235 studies. An increasing number of articles in the literature have indicated that a correlation 236 between systematic inflammatory response and acute mountain sickness (AMS) exists. The 237 reason may be that hypoxia was shown to alter cellular immunity and cytokine release. There is pulmonary vascular remodeling and is possibly responsible for the occurrence of high-altitude diseases (Savale et al. 2009). Synthesis of IL-6 is stimulated by TNF and IL-1, which has been identified as a critical mediator of inflammation in tissues ( $\mathrm{Li}$ et al. 2015).

Boos et al. reported that an increase in cytokines, such as IL-6 and IL-17 $\alpha$, was correlated with exercise at high altitudes(Boos et al. 2016). In addition, $\mathrm{Lu}$ et al. identified four cytokines including IGFBP6 (insulin-like growth factor binding protein 6), Dkk4 (dickkopf WNT signaling pathway inhibitor 4), SAA1 (serum amyloid A1), and IL-17RA (interleukin 17 receptor A), which might predict AMS susceptibility in a low-altitude environment (Lu et al. 2016). Liu et al. found IL-10 dysregulation, which is involved in immune and inflammatory responses, in AMS through transcriptome analysis. The reduction of IL-10 after exposure to high altitude was strongly correlated with AMS (Liu et al. 2017). In addition to the proinflammatory cytokines, specific acute phase proteins have also been demonstrated to be changed under the condition of high altitude. Hypobaric hypoxia is a pathophysiological condition triggering the disturbance of cell organization, leading to protein, lipid, or DNA damage through oxidative acute mountain sickness stress (Singh et al. 2010).

In addition to causing symptoms of AMS, inflammation may play a key role in increasing ventilation, driven by increased chemosensitivity of the carotid body, which accompanies acclimatization to high altitude. A number of studies have documented increased cytokines and cytokine gene expression in the carotid body during exposure to acute or chronic hypoxia (Lam et al. 2012b). Consistent with this, administration of anti-inflammatory drugs prevents increased cytokine expression and increased carotid body chemosensitivity in animals. A number of previous studies have documented increases in proinflammatory cytokines at high altitude, including IL-6 (Klausen et al. 1997). Furthermore, higher levels of interleukin receptor antagonist (IL-RA), a highly competitive antagonist of the proinflammatory cytokine IL-1, have been found in AMS-resistant subjects but not in AMS-sensitive subjects (Julian et al. 2011). However, there is no consensus on the role of inflammation in high-altitude acclimatization. Some argue that the elevation of cytokines occurs after the onset of AMS, and thus the time course of the inflammatory response contradicted the claim that inflammation plays a role in AMS (Hartmann et al. 2000). Furthermore, an older study shows unchanged concentrations of proinflammatory cytokines in response to hypoxia (Swenson et al. 1997). However, we find overexpression of the IL-17, TNF and chemokine signaling pathways in the liver under hypobaric hypoxia.

Notably, mineral absorption and galactose metabolish pathways were also over-represented. Concerning the galactose metabolism, most of the galactose enters the liver of rat, where it is mainly converted to glucose, which is then either incorporated into glycogen or used for energy, 
274

275

276

277

278

279

280

281

282

283

284

285

286

287

288

289

290

291

292

293

294

295

296

\section{7}

298

299

300

301

302

303

304

305

306

307

308

309

310

311

312

connected with glycolysis. It seems that at the hypobaric hypoxia exposure, rats acquire more energy than as usual to survive. As far as we know, no study about the galactose metabolism in the hypobaric hypoxia condition was reported, though glycolysis was identified before (Baze et al. 2010). The bone resorption and bone material properties are affected by the hypobaric hypoxia environment (Bozzini et al. 2009; Guner et al. 2013). Obviously, mineral absorption, induced by the hypobaric hypoxia, played critical roles in the bone resorption and bone mineral density. It indicates that the metabolism in rat is perturbed by the hypobaric hypoxia condition. These responses in metabolism are probably a way to adapt to the hypobaric hypoxia environment.

Pathway analysis for each co-expression cluster shows that the cytokine-cytokine receptor interaction pathway and JAK-STAT signaling pathway were enriched only in the first cluster. Dysregulation of the JAK -STAT pathways leads to hematopoietic and immune diseases. The JAK2-STAT pathways have been reported to be involved in cell protection and injury. The JAK2 inhibitor and overexpression of its dominant negative JAK2 protein improve cell survival against peroxide and superoxide anions. Inactivation of JAK2 has been shown to be a potential method in endothelial cells to avoid oxidative stress-induced death (Neria et al. 2009).

Parthenolide has been reported to inhibit JAK1 and STAT3 activity. The product of reactive oxygen species (ROS) inhibits the STAT3 signaling pathway by targeting JAK1 (Kurdi \& Booz 2007).

Limitations of our study include the number of subjects, which could not generate precise cutoff values for AMS diagnosis in rats. In addition, we were not able to obtain blood samples in weeks 2 and 4; thus, we were unable to make a comparison and observe whether the changes in these indices are consistent with plasma.

\section{CONCLUSIONS}

In conclusion, this study further verifies an increase in immune-related pathways in the first 2 weeks of high altitude exposure, but not in week 4. Our research found immune-related and metabolic pathways, such as cytokine-cytokine receptor interaction and galactose metabolism, were highly enriched in the KEGG pathway analysis for hypobaric hypoxia. Similar results were found in the Gene Ontology analysis. Cogena analysis showed that immune-related pathways were mainly upregulated and enriched for acute hypobaric hypoxia ( 2 week). It may be an important physiological cue in order for the rats to respond to a stressful environment. More studies are necessary to reveal the underlying molecular mechanisms in the liver controlling the expressions of key enzymes involved in the process.

\section{References}

Akopian NS, Sarkisian NV, Adamian N, Bagdasarian KV, and Akopian AN. 2002. [Pathological types of breathing during acute hypoxia]. Aviakosm Ekolog Med 36:32-37.

Bailey D, Kleger G, Holzgraefe M, Ballmer P, and Bärtsch P. 2004. Pathophysiological significance of peroxidative stress, neuronal damage, and membrane permeability in acute mountain sickness. J Appl Physiol 96:14591463.

Peer) reviewing PDF | (2018:10:31961:1:3:ACCEPTED 14 Jan 2019) 
Baze MM, Schlauch K, and Hayes JP. 2010. Gene expression of the liver in response to chronic hypoxia. Physiol Genomics 41:275-288. 10.1152/physiolgenomics.00075.2009

Bigham AW, and Lee FS. 2014. Human high-altitude adaptation: forward genetics meets the HIF pathway. Genes Dev 28:2189-2204. 10.1101/gad.250167.114

Bonewald LF. 1999. Regulation and regulatory activities of transforming growth factor beta. Crit Rev Eukaryot Gene Expr 9:33-44.

Boos CJ, Woods DR, Varias A, Biscocho S, Heseltine P, and Mellor AJ. 2016. High Altitude and Acute Mountain Sickness and Changes in Circulating Endothelin-1, Interleukin-6, and Interleukin-17a. High Alt Med Biol 17:25-31. 10.1089/ham.2015.0098

Bosco MC, Puppo M, Santangelo C, Anfosso L, Pfeffer U, Fardin P, Battaglia F, and Varesio L. 2006. Hypoxia modifies the transcriptome of primary human monocytes: modulation of novel immune-related genes and identification of CC-chemokine ligand 20 as a new hypoxia-inducible gene. J Immunol 177:1941-1955.

Bozzini C, Olivera MI, Huygens P, Alippi RM, and Bozzini CE. 2009. Long-term exposure to hypobaric hypoxia in rat affects femur cross-sectional geometry and bone tissue material properties. Ann Anat 191:212-217. 10.1016/j.aanat.2008.11.002

Dosek A, Ohno H, Acs Z, Taylor A, and Radak Z. 2007. High altitude and oxidative stress. Respir Physiol Neurobiol 158:128-131.

Guner I, Uzun DD, Yaman MO, Genc H, Gelisgen R, Korkmaz GG, Hallac M, Yelmen N, Sahin G, Karter Y, and Simsek G. 2013. The effect of chronic long-term intermittent hypobaric hypoxia on bone mineral density in rats: role of nitric oxide. Biol Trace Elem Res 154:262-267. 10.1007/s12011-013-9722-8

Guo G, Zhu G, Sun W, Yin C, Ren X, Wang T, and Liu M. 2014. Association of arterial oxygen saturation and acute mountain sickness susceptibility: a meta-analysis. Cell Biochem Biophys 70:1427-1432. 10.1007/s12013-014-0076-4

Hartmann G, Tschop M, Fischer R, Bidlingmaier C, Riepl R, Tschop K, Hautmann H, Endres S, and Toepfer M. 2000. High altitude increases circulating interleukin-6, interleukin-1 receptor antagonist and C-reactive protein. Cytokine 12:246-252. 10.1006/cyto.1999.0533

Hochachka PW, Buck LT, Doll CJ, and Land SC. 1996. Unifying theory of hypoxia tolerance: molecular/metabolic defense and rescue mechanisms for surviving oxygen lack. Proc Natl Acad Sci U S A 93:9493-9498.

Honigman B, Theis MK, Koziol-McLain J, Roach R, Yip R, Houston C, Moore LG, and Pearce P. 1993. Acute mountain sickness in a general tourist population at moderate altitudes. Ann Intern Med 118:587-592.

Horscroft JA, Kotwica AO, Laner V, West JA, Hennis PJ, Levett DZH, Howard DJ, Fernandez BO, Burgess SL, Ament Z, Gilbert-Kawai ET, Vercueil A, Landis BD, Mitchell K, Mythen MG, Branco C, Johnson RS, Feelisch M, Montgomery HE, Griffin JL, Grocott MPW, Gnaiger E, Martin DS, and Murray AJ. 2017. Metabolic basis to Sherpa altitude adaptation. Proc Natl Acad Sci U S A 114:6382-6387. $10.1073 /$ pnas. 1700527114

Ivanova H, Vervliet T, Missiaen L, Parys JB, De Smedt H, and Bultynck G. 2014. Inositol 1,4,5-trisphosphate receptor-isoform diversity in cell death and survival. Biochim Biophys Acta 1843:2164-2183. 10.1016/j.bbamcr.2014.03.007

Jia Z, Liu Y, Guan N, Bo X, Luo Z, and Barnes M. 2016. Cogena, a novel tool for co-expressed gene-set enrichment analysis, applied to drug repositioning and drug mode of action discovery. BMC Genomics 17:414.

Julian CG, Subudhi AW, Wilson MJ, Dimmen AC, Pecha T, and Roach RC. 2011. Acute mountain sickness, 
354

355

356

357

358

359

360

361

362

363

364

365

366

367

368

369

370

371

372

373

374

375

376

377

378

379

380

381

382

383

384

385

386

387

388

389

390

391

392

393

394

inflammation, and permeability: new insights from a blood biomarker study. J Appl Physiol (1985) 111:392-399. 10.1152/japplphysiol.00391.2011

Jung C, Sörensson P, Saleh N, Arheden H, Rydén L, and Pernow J. 2012. Circulating endothelial and platelet derived microparticles reflect the size of myocardium at risk in patients with ST-elevation myocardial infarction. Atherosclerosis 221:226-231.

Kaelin WG, Jr., and Ratcliffe PJ. 2008. Oxygen sensing by metazoans: the central role of the HIF hydroxylase pathway. Mol Cell 30:393-402. 10.1016/j.molcel.2008.04.009

Klausen T, Olsen NV, Poulsen TD, Richalet JP, and Pedersen BK. 1997. Hypoxemia increases serum interleukin-6 in humans. Eur J Appl Physiol Occup Physiol 76:480-482. 10.1007/s004210050278

Kurdi M, and Booz G. 2007. Evidence that IL-6-type cytokine signaling in cardiomyocytes is inhibited by oxidative stress: parthenolide targets JAK1 activation by generating ROS. J Cell Physiol 212:424-431.

Lam S, Liu Y, Ng K, Lau C, Liong E, Tipoe G, and Fung M. 2012a. Chronic intermittent hypoxia induces local inflammation of the rat carotid body via functional upregulation of proinflammatory cytokine pathways. Histochem Cell Biol 137:303-317.

Lam SY, Liu Y, Ng KM, Lau CF, Liong EC, Tipoe GL, and Fung ML. 2012b. Chronic intermittent hypoxia induces local inflammation of the rat carotid body via functional upregulation of proinflammatory cytokine pathways. Histochem Cell Biol 137:303-317. 10.1007/s00418-011-0900-5

LG W, Y H, and QY H. 2012. clusterProfiler: an R package for comparing biological themes among gene clusters.\%A Yu G. Omics : a journal of integrative biology 16:284-287.

Li G, Fu J, Zhao Y, Ji K, Luan T, and Zang B. 2015. Alpha-lipoic acid exerts anti-inflammatory effects on lipopolysaccharide-stimulated rat mesangial cells via inhibition of nuclear factor kappa B (NF-kappaB) signaling pathway. Inflammation 38:510-519. 10.1007/s10753-014-9957-3

Liu B, Chen J, Zhang L, Gao Y, Cui J, Zhang E, Xu G, Liang Y, Liang Y, Wang J, and Gao Y. 2017. IL-10 Dysregulation in Acute Mountain Sickness Revealed by Transcriptome Analysis. Front Immunol 8:628. 10.3389/fimmu.2017.00628

Lu H, Wang R, Li W, Xie H, Wang C, Hao Y, Sun Y, and Jia Z. 2016. Plasma cytokine profiling to predict susceptibility to acute mountain sickness. Eur Cytokine Netw 27:90-96. 10.1684/ecn.2016.0383

Murray AJ. 2016. Energy metabolism and the high-altitude environment. Exp Physiol 101:23-27. 10.1113/EP085317

Neria F, Castilla M, Sanchez R, Gonzalez Pacheco F, Deudero J, Calabia O, Tejedor A, Manzarbeitia F, Ortiz A, and Caramelo C. 2009. Inhibition of JAK2 protects renal endothelial and epithelial cells from oxidative stress and cyclosporin A toxicity. Kidney Int 75:227-234.

Ni Q, Shao Y, Wang YZ, Jing YH, and Zhang YC. 2014. Impact of high altitude on the hepatic fatty acid oxidation and synthesis in rats. Biochem Biophys Res Commun 446:574-579. 10.1016/j.bbrc.2014.03.001

O'Connor T, Dubowitz G, and Bickler PE. 2004. Pulse oximetry in the diagnosis of acute mountain sickness. High Alt Med Biol 5:341-348. 10.1089/ham.2004.5.341

Pompella A, and Corti A. 2015. Editorial: the changing faces of glutathione, a cellular protagonist. Front Pharmacol 6:98.

Roach RC, Hackett PH, Oelz O, Bartsch P, Luks AM, MacInnis MJ, and Baillie JK. 2018. The 2018 Lake Louise Acute Mountain Sickness Score. High Alt Med Biol 19:4-6. 10.1089/ham.2017.0164

Savale L, Tu L, Rideau D, Izziki M, Maitre B, Adnot S, and Eddahibi S. 2009. Impact of interleukin-6 on hypoxia-

Peer) reviewing PDF | (2018:10:31961:1:3:ACCEPTED 14 Jan 2019) 
induced pulmonary hypertension and lung inflammation in mice. Respir Res 10:6. 10.1186/1465-9921-10-6 Seys S, Daenen M, Dilissen E, Van Thienen R, Bullens D, Hespel P, and Dupont L. 2013. Effects of high altitude and cold air exposure on airway inflammation in patients with asthma. Thorax 68:906-913.

Singh M, Shukla D, Thomas P, Saxena S, and Bansal A. 2010. Hypoxic preconditioning facilitates acclimatization to hypobaric hypoxia in rat heart. J Pharm Pharmacol 62:1729-1739. 10.1111/j.2042-7158.2010.01163.x

Swenson ER, MacDonald A, Vatheuer M, Maks C, Treadwell A, Allen R, and Schoene RB. 1997. Acute mountain sickness is not altered by a high carbohydrate diet nor associated with elevated circulating cytokines. Aviat Space Environ Med 68:499-503.

Taylor CT. 2008. Interdependent roles for hypoxia inducible factor and nuclear factor-kappaB in hypoxic inflammation. J Physiol 586:4055-4059. 10.1113/jphysiol.2008.157669

Walmsley SR, Print C, Farahi N, Peyssonnaux C, Johnson RS, Cramer T, Sobolewski A, Condliffe AM, Cowburn AS, Johnson N, and Chilvers ER. 2005. Hypoxia-induced neutrophil survival is mediated by HIF-1alphadependent NF-kappaB activity. $J$ Exp Med 201:105-115. 10.1084/jem.20040624

Wang C, Jiang H, Duan J, Chen J, Wang Q, Liu X, and Wang C. 2018. Exploration of Acute Phase Proteins and Inflammatory Cytokines in Early Stage Diagnosis of Acute Mountain Sickness. High Alt Med Biol 19:170177.

Ward M. 1954. High altitude deterioration. Proc R Soc Lond B Biol Sci 143:40-42.

Weil WM, Glassner PJ, and Bosco JA, 3rd. 2007. High-altitude illness and muscle physiology. Bull NYU Hosp Jt Dis 65:72-77.

Wood J, Johnson J, Mattioli L, and Gonzalez N. 2000. Systemic hypoxia increases leukocyte emigration and vascular permeability in conscious rats. $J$ Appl Physiol 89:1561-1568.

Yu G, Wang LG, Han Y, and He QY. 2012. clusterProfiler: an R package for comparing biological themes among gene clusters. OMICS 16:284-287. 10.1089/omi.2011.0118 
Figure $\mathbf{1}$ (on next page)

Heatmap of the 497 DEGs in the three groups.

Colors represent the normalized gene expression values of DEGs. The control $L$ group is separated with the hypobaric hypoxia groups. The W4L group is closer with the L group than with $W 2 L$ group. The last number in the group labels represent sample id in this group. 
Color Key

and Histogram PeerJ
Manuscript to be reviewed
흥워

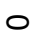

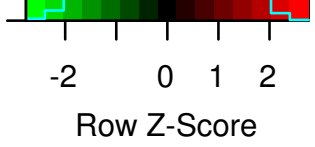

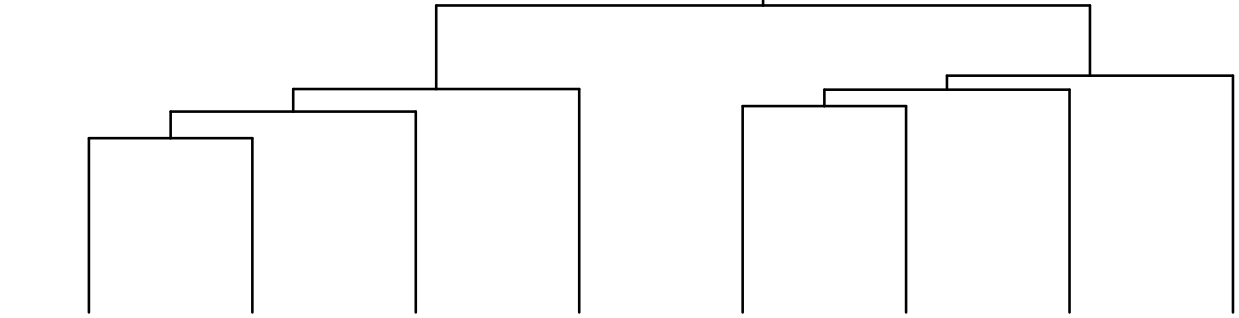
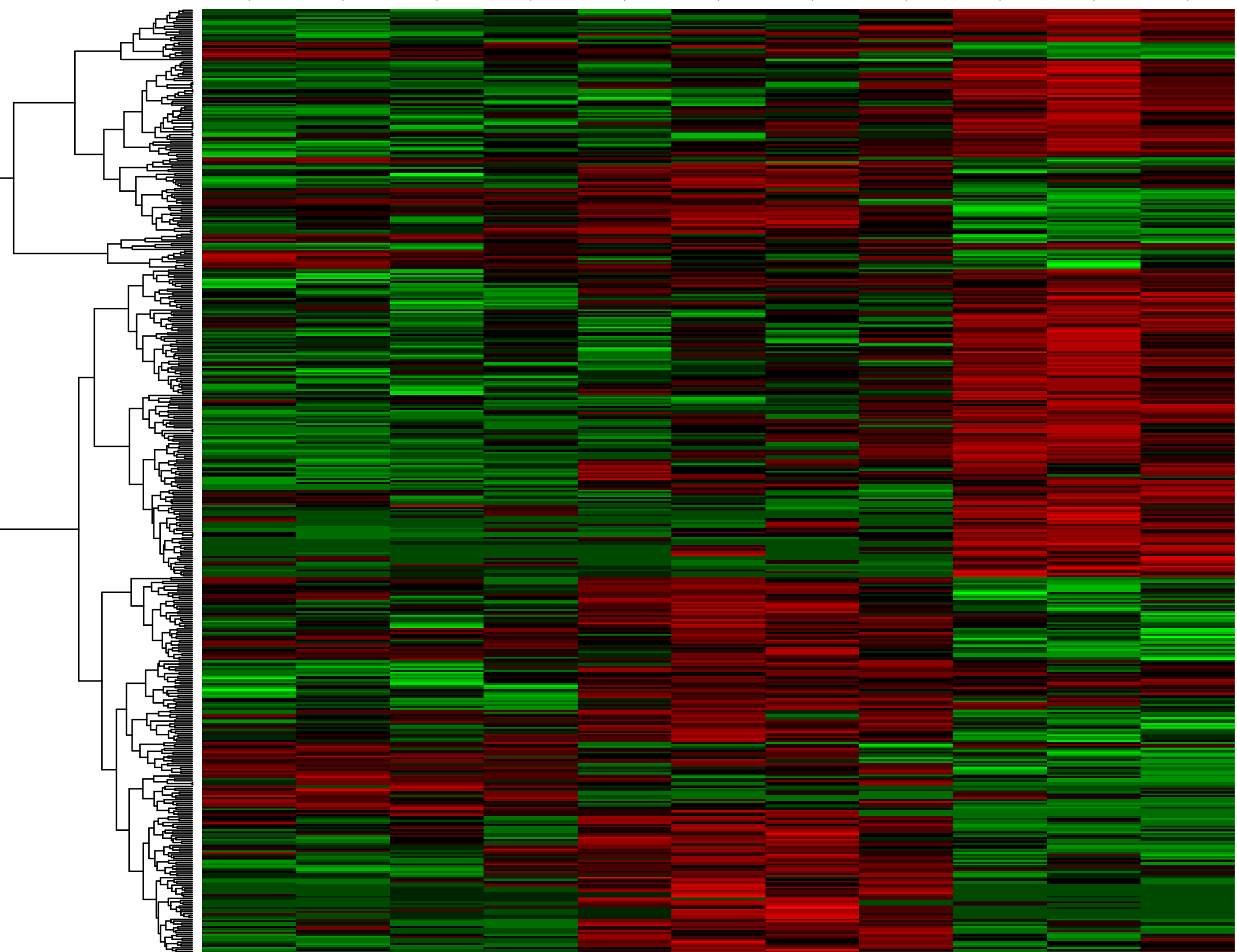

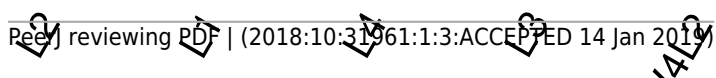

$2 \sqrt{x}$
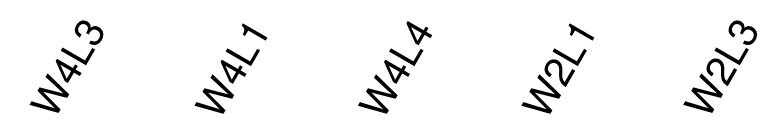

$s^{2}$ 
Figure 2 (on next page)

Principal component analysis of the samples in the three groups.

The three groups are clearly separated. 


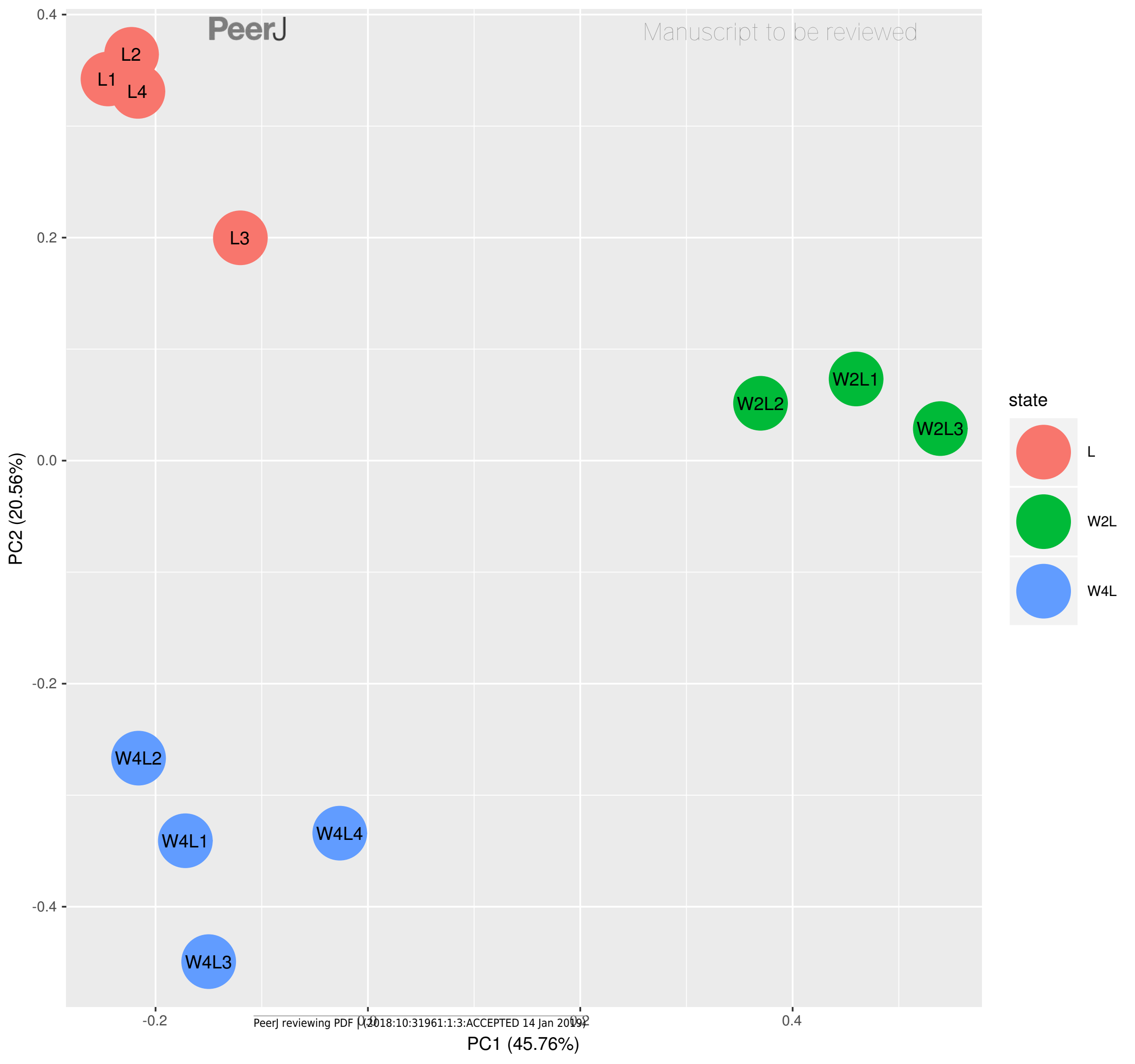


Figure 3 (on next page)

Pathway analysis of the DEGs.

The top 8 significant KEGG pathways are shown. 
Cytokine-cytokine receptor interaction

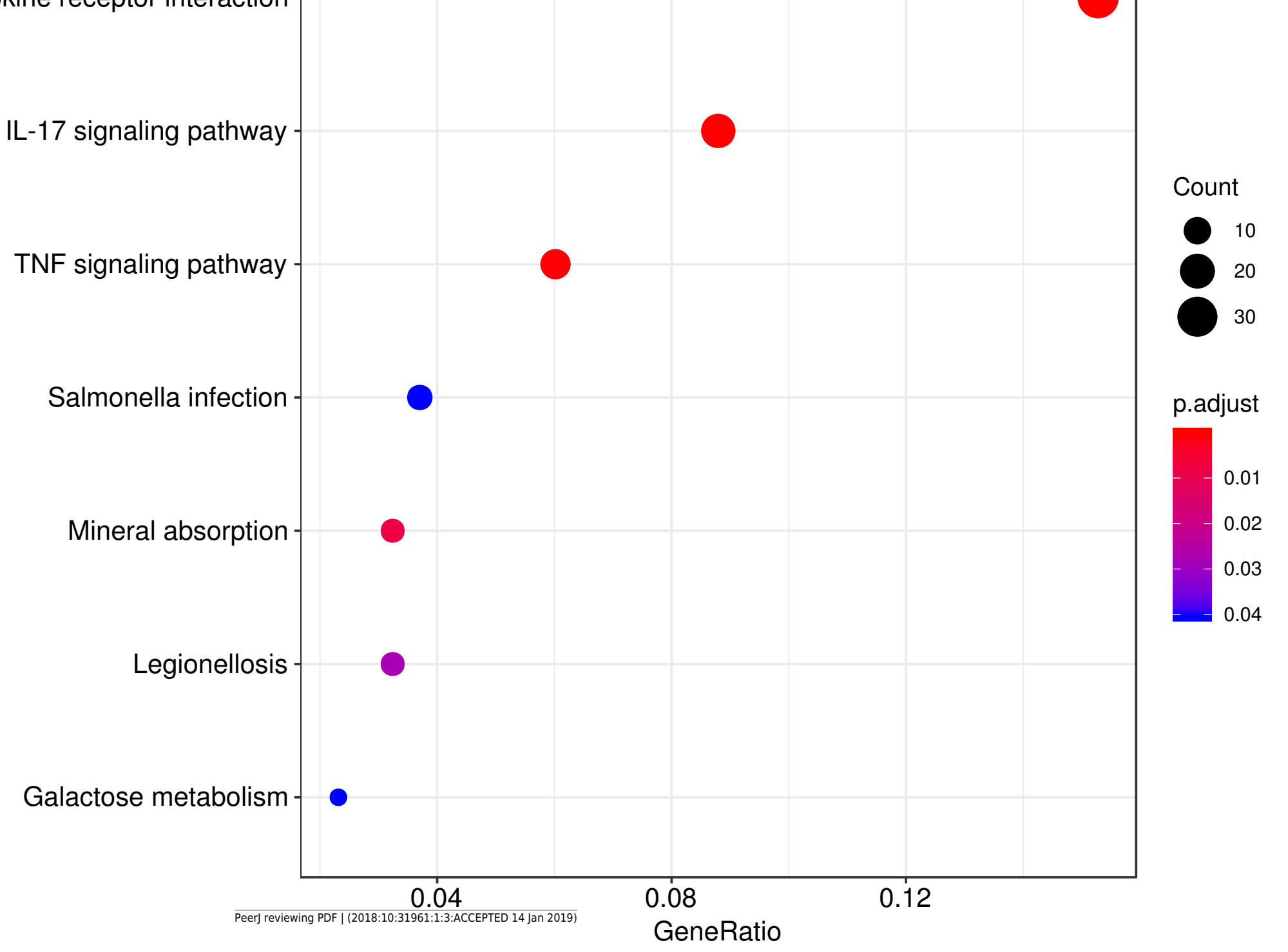


Figure 4 (on next page)

GO analysis results from hypobaric hypoxia.

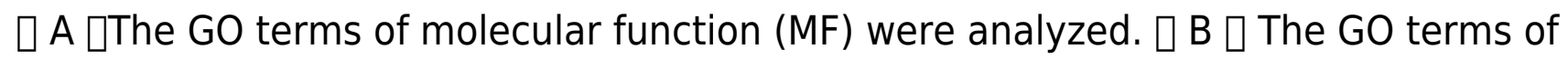
biological process (BP) were analyzed. $\square \mathrm{C} \square$ The GO terms of cellular component (CC) 


\section{PeerJ}

leukocyte migration response to tumor necrosis factor myeloid leukocyte migration leukocyte chemotaxis granulocyte migration response to interleukin-1 granulocyte chemotaxis

neutrophil migration cellular response to interleukin-1 neutrophil chemotaxis

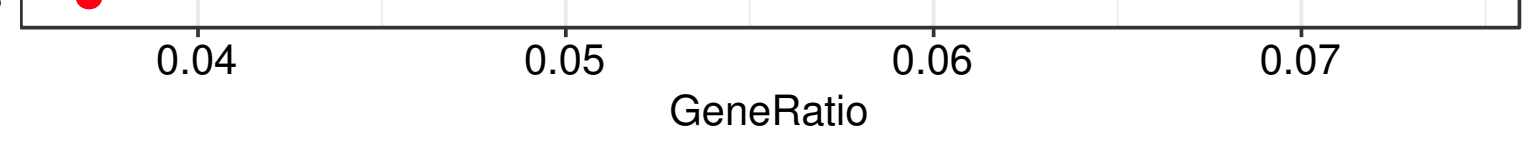

\section{GO_MF}

receptor regulator activity receptor ligand activity G-protein coupled receptor binding cytokine activity cytokine receptor binding organic acid binding carbohydrate binding carboxylic acid binding chemokine activity chemokine receptor binding

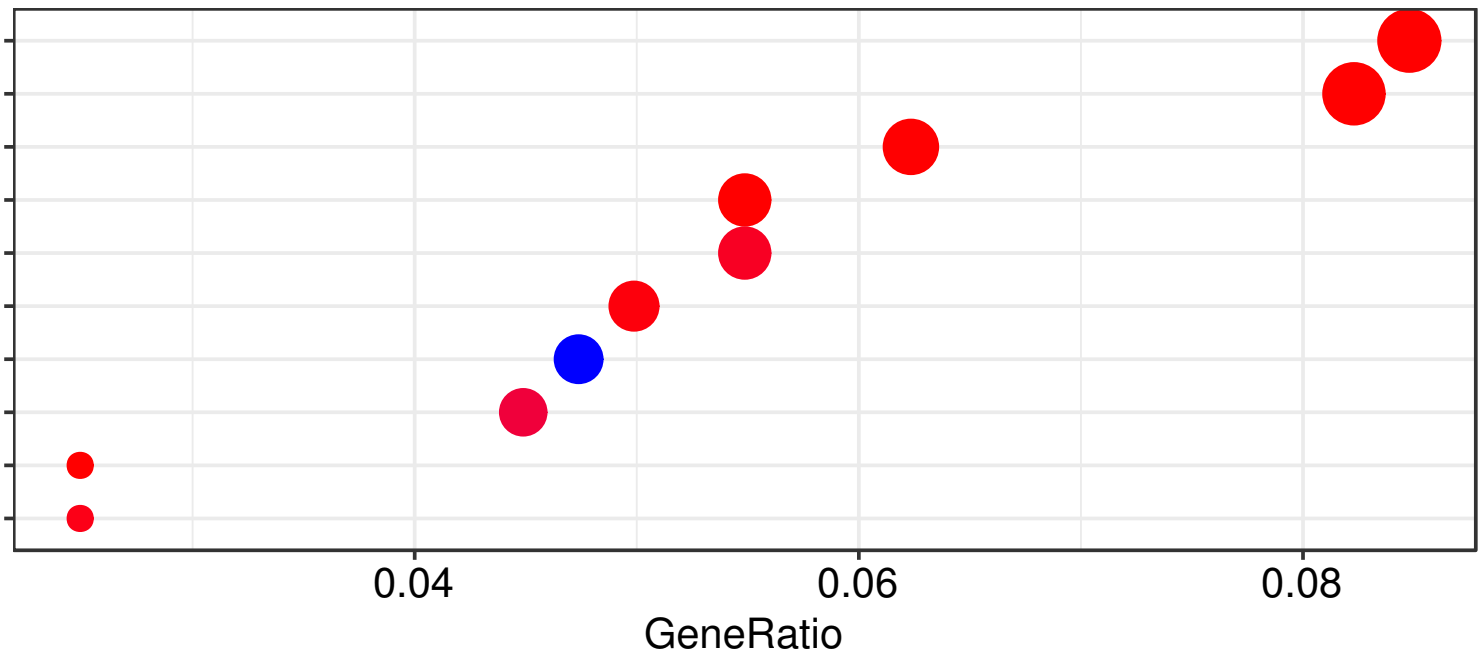

GO_CC

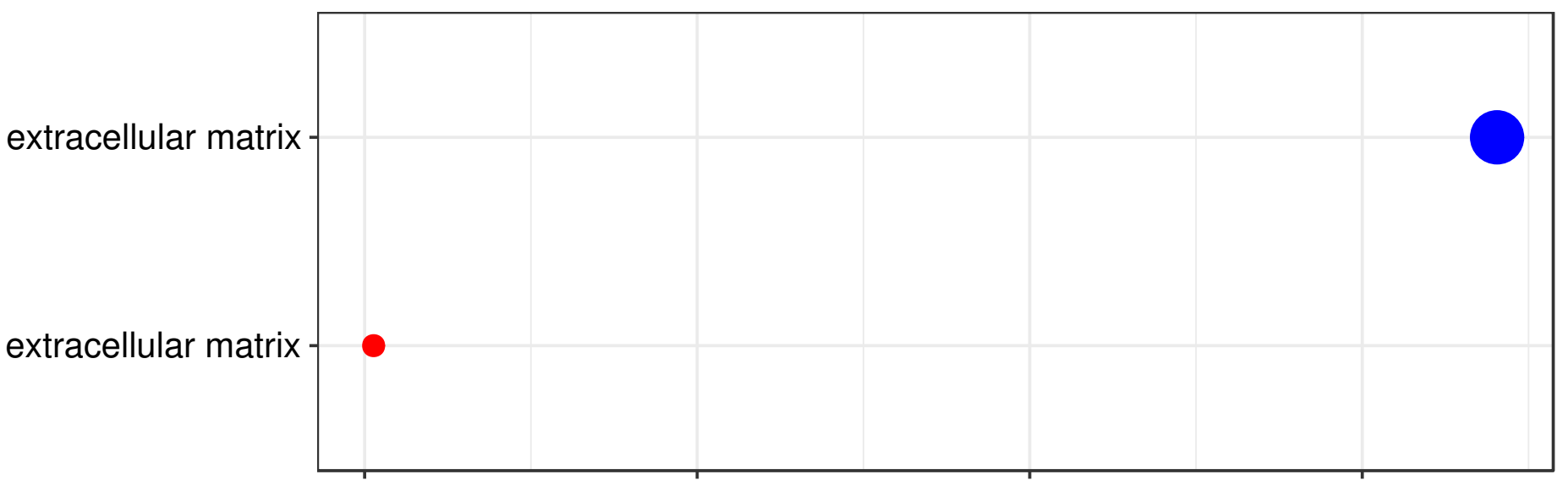

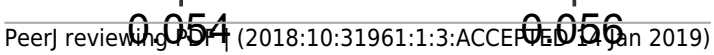


Figure $\mathbf{5}$ (on next page)

Heatmap of co-expressed genes.

Kmeans clustering method and 3 cluster are shown in the heatmap, plotted with the cogena Bioconductor package. 
Figure 6 (on next page)

Co-expression pathway analysis.

KEGG pathways are shown with the enrichment score based on the cogena package. 
kegg_cytokine_cytokine_receptor_interaction -

kegg_nod_like_receptor_signaling_pathway - 